\title{
Prescriptions for Correcting Ultraviolet-based Redshifts for Luminous Quasars at High Redshift
}

\author{
Cooper Dix ${ }^{1}$ (1), Ohad Shemmer ${ }^{1}$ (i), Michael S. Brotherton ${ }^{2}$ (1), Richard F. Green ${ }^{3}$, Michelle Mason ${ }^{2}$ (1), and Adam D. Myers ${ }^{2}$ \\ ${ }^{1}$ Department of Physics, University of North Texas, Denton, TX 76203, USA; cooperdix@ my.unt.edu \\ ${ }^{2}$ Department of Physics and Astronomy, University of Wyoming, Laramie, WY 82071, USA \\ 3 Department of Astronomy, University of Arizona, Tucson, AZ 85721, USA \\ Received 2019 October 31; revised 2020 January 21; accepted 2020 February 17; published 2020 April 8
}

\begin{abstract}
High-redshift quasars typically have their redshift determined from rest-frame ultraviolet (UV) emission lines. However, these lines, and more specifically the prominent C IV $\lambda 1549$ emission line, are typically blueshifted yielding highly uncertain redshift estimates compared to redshifts determined from rest-frame optical emission lines. We present near-infrared spectroscopy of 18 luminous quasars at $2.15<z<3.70$ that allows us to obtain reliable systemic redshifts for these sources. Together with near-infrared spectroscopy of an archival sample of 44 quasars with comparable luminosities and redshifts, we provide prescriptions for correcting UV-based redshifts. Our prescriptions reduce velocity offsets with respect to the systemic redshifts by $\sim 140 \mathrm{~km} \mathrm{~s}^{-1}$ and reduce the uncertainty on the UV-based redshift by $\sim 25 \%$ with respect to the best method currently used for determining such values. We also find that the redshifts determined from the Sloan Digital Sky Survey Pipeline for our sources suffer from significant uncertainties, which cannot be easily mitigated. We discuss the potential of our prescriptions to improve UV-based redshift corrections given a much larger sample of high-redshift quasars with near-infrared spectra.
\end{abstract}

Unified Astronomy Thesaurus concepts: Galaxy distances (590); Quasars (1319); Active galactic nuclei (16)

\section{Introduction}

The best practical indicators for a quasar's systemic redshift $\left(z_{\text {sys }}\right)$ lie in the rest-frame optical band, particularly the prominent [O III] $\lambda 5007, \mathrm{Mg}$ II $\lambda 2800$, and the Balmer emission lines (e.g., Boroson 2005; Shen et al. 2016). However, at high-redshift $(z \gtrsim 0.8), \approx 10^{5}$ quasars typically have their $z_{\text {sys }}$ values determined from rest-frame ultraviolet (UV) spectra since only $0.1 \%$ of these quasars have corresponding rest-frame optical information from near-infrared (NIR) spectra (e.g., Schneider et al. 2010; Pâris et al. 2017, 2018). Unfortunately, the UV-based $z_{\text {sys }}$ estimates are highly inaccurate and imprecise given that the UV emission lines are usually blueshifted by up to $\approx 3000 \mathrm{~km} \mathrm{~s}^{-1}$ (e.g., Gaskell 1982; Tytler \& Fan 1992; Gibson et al. 2009; Shen et al. 2016). Mitigating these biases requires identifying robust corrections to UVbased redshifts.

Reliable redshift estimates are needed for multiple reasons. For example, accurate redshift estimates provide information on the kinematics of the outflowing material in the vicinity of a supermassive black hole, which likely impacts the star formation rate in the quasar's host galaxy (e.g., Hopkins \& Elvis 2010). Additionally, various cosmological studies utilize conversions between redshift differences and distances (e.g., Hogg 1999; Zhao et al. 2019). In this context, a velocity offset of $500 \mathrm{~km} \mathrm{~s}^{-1}$ corresponds to a comoving distance of $\approx 5 h^{-1} \mathrm{Mpc}$ at $z=2.5$, which can impact our understanding of, e.g., quasar clustering as velocity offsets can be misinterpreted to be distances in the redshift direction (e.g., FontRibera et al. 2013; Prochaska et al. 2013).

The Sloan Digital Sky Survey (SDSS; York et al. 2000) provides observed-frame optical spectra and redshifts for hundreds of thousands of quasars. The redshifts determined for these quasars stem from a cross-correlation by a composite quasar template spectrum provided by Vanden Berk et al. (2001).
However, these estimates become increasingly uncertain in highredshift quasars because mostly rest-frame UV emission lines are present in the optical band. The first meaningful correction to these UV-based redshifts was achieved by Hewett \& Wild (2010, hereafter HW10). They achieved this by introducing a two-part linear relation between the absolute magnitude and redshift of quasars. A more recent improvement to the HW10 method was achieved by Mason et al. (2017, hereafter M17), by comparing [O III]-based $z_{\text {sys }}$ values with the spectral properties of the C IV $\lambda 1549$ emission line for 45 quasars with $z \gtrsim 2.2$.

In this work, we expand on the M17 method by adding highquality NIR spectra of 18 quasars at $2.15<z<3.70$. We perform multiple regression analyses and provide improved prescriptions for correcting a variety of UV-based redshifts when the C IV line is available in the spectrum. This paper is organized as follows. In Section 2, we describe our sample selection, observations, and data analysis. In Section 3, we present our spectroscopic measurements, and in Section 4 we discuss our results. Our conclusions are presented in Section 5. Throughout this paper, we compute luminosity distances using $H_{0}=70 \mathrm{~km} \mathrm{~s}^{-1} \mathrm{Mpc}^{-1}, \Omega_{\mathrm{M}}=0.3$, and $\Omega_{\Lambda}=0.7$ (e.g., Spergel et al. 2007).

\section{Sample Selection, Observations, and Data Analysis}

We have selected a sample of 18 quasars for our investigation based upon the following criteria:

1. Availability of a flux-calibrated optical spectrum from the SDSS recorded in the Data Release 10 quasar catalog (Pâris et al. 2014).

2. Brightness in the range $m_{i}<18.5$ in order to keep the signal-to-noise ratio $(\mathrm{S} / \mathrm{N})$ of the $\mathrm{H} \beta$ region of the respective NIR spectrum, obtained with a $3.8 \mathrm{~m}$ telescope, at $\approx 40$. 
Table 1

Observation Log

\begin{tabular}{|c|c|c|c|c|c|c|c|}
\hline $\begin{array}{l}\text { Quasar } \\
\text { (1) }\end{array}$ & $\begin{array}{c}z \\
(2)\end{array}$ & $\begin{array}{l}z_{\text {ref }}^{a} \\
\text { (3) }\end{array}$ & $\begin{array}{c}z_{\text {sys }}^{\mathrm{b}} \\
(4)\end{array}$ & $\begin{array}{c}H^{\mathrm{c}} \\
(\mathrm{mag}) \\
(5)\end{array}$ & $\begin{array}{c}K^{\mathrm{c}} \\
(\mathrm{mag}) \\
(6)\end{array}$ & $\begin{array}{l}\text { Observation } \\
\text { Date } \\
\text { (7) }\end{array}$ & $\begin{array}{c}\text { Net Exposure } \\
\text { (s) } \\
\text { (8) }\end{array}$ \\
\hline SDSS J013435.67-093102.9 & 2.225 & 1 & 2.214 & 14.8 & 13.6 & 2016 Aug 25 & 2880 \\
\hline SDSS J014850.64-090712.8 & 3.303 & 1 & 3.329 & 16.7 & 15.5 & 2016 Sep 19 & 4800 \\
\hline SDSS J073607.63+220758.9 & 3.464 & 2 & 3.445 & 16.1 & 14.9 & 2016 Sep 20 & 3840 \\
\hline$\ldots$ & $\ldots$ & $\ldots$ & .. & $\ldots$ & $\ldots$ & 2016 Sep 22 & 3840 \\
\hline SDSS J142243.02+441721.2 & 3.530 & 1 & $3.651^{\mathrm{e}}$ & 15.2 & 14.4 & 2016 Sep 7 & 1920 \\
\hline SDSS J153750.10+201035.7 & 3.413 & 3 & 3.413 & 15.7 & 15.4 & 2016 Sep 22 & 3840 \\
\hline SDSS J153830.55+085517.0 & 3.563 & 1 & 3.550 & 15.6 & 14.6 & 2016 Sep 19 & 1920 \\
\hline SDSS J154359.43+535903.1 ${ }^{\mathrm{d}}$ & 2.379 & 1 & 2.364 & 15.0 & 14.2 & 2016 Sep 21 & 2880 \\
\hline SDSS J154446.33+412035.7 & 3.551 & 1 & $3.567^{\mathrm{e}}$ & 15.6 & 15.5 & 2016 Sep 20 & 3840 \\
\hline SDSS J154938.71+124509.1 & 2.377 & 4 & 2.369 & 14.5 & 13.5 & 2016 Sep 5 & 1920 \\
\hline SDSS J155013.64+200154.5 & 2.196 & 1 & 2.188 & 15.1 & 14.2 & 2016 Sep 19 & 2400 \\
\hline SDSS J160222.72+084538. $4^{\mathrm{d}}$ & 2.276 & 1 & 2.275 & 15.0 & 14.0 & 2016 Sep 6 & 2880 \\
\hline SDSS J163300.13+362904. $8^{\mathrm{d}}$ & 3.575 & 1 & 3.570 & 15.5 & 15.1 & 2016 Sep 22 & 2640 \\
\hline SDSS J165137.52+400218.9 & 2.342 & 1 & 2.338 & 15.0 & 13.7 & 2016 Sep 6 & 2880 \\
\hline SDSS J172237.85+385951.8 & 3.390 & 2 & 3.367 & 16.0 & 15.3 & 2016 Sep 19 & 3840 \\
\hline SDSS J210524.47+000407.3 & 2.307 & 1 & $2.344^{\mathrm{e}}$ & 14.7 & 13.8 & 2016 Aug 26 & 1920 \\
\hline SDSS J212329.46-005052.9 & 2.268 & 1 & $2.270^{\mathrm{f}}$ & 14.6 & 13.9 & 2016 Sep 5 & 1920 \\
\hline SDSS J221506.02+151208.5 & 3.285 & 2 & 3.284 & 16.4 & 15.2 & 2016 Aug 26 & 3840 \\
\hline SDSS J235808.54+012507.2 & 3.401 & 2 & 3.389 & 14.7 & 13.8 & 2016 Aug 26 & 2880 \\
\hline
\end{tabular}

Notes.

a (1) HW10; (2) Chen et al. (2014), (3) Richards et al. (2009), (4) Hutchings et al. (2006).

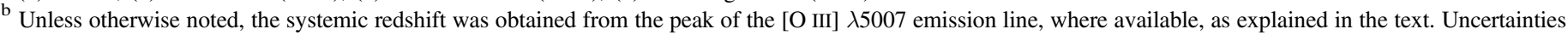
on these values, discussed in Section 2.1 , average $\sim 150 \mathrm{~km} \mathrm{~s}^{-1}$.

${ }^{c}$ Vega-based magnitudes were obtained from 2MASS.

d Indicates a BAL quasar.

e Systemic redshift was determined from $\lambda_{\text {peak }}$ of the $\mathrm{H} \beta$ emission line.

${ }^{\mathrm{f}}$ Systemic redshift was determined from $\lambda_{\text {peak }}$ of the Mg II emission line from the SDSS spectrum of the source.

3. Redshift within one of the following intervals, $2.15<z<2.65^{4}$ and $3.20<z<3.70$, in which, at a minimum, the $\mathrm{H} \beta$ and [O III] lines can be modeled accurately within one of the NIR transmission windows in the $H$ or $K$ bands.

Spectroscopic observations of this sample were performed at the United Kingdom Infrared Telescope (UKIRT) on Maunakea, Hawaii. The observation log and quasar basic properties appear in Table 1.

We utilized the UKIRT Imager-Spectrometer with a slit width of 0 !" 24 to maximize the resolution at the expense of potentially higher slit losses. During these observations, the telescope was nodded in an ABBA pattern in order to obtain primary background subtraction. The broad band $\mathrm{B} 2$ filter was used in order to obtain a wavelength range of approximately $1.395-2.506 \mu \mathrm{m}$, spanning the $H$ and $K$ bands as necessary. The dispersion for these observations was $10.9 \AA$ pixel $^{-1}$ with a spectral resolution of $R \sim 448$. Standard stars of spectral type $\mathrm{G}$ and $\mathrm{F}$ were observed on each night alongside the quasar in order to remove the telluric features that are present in the quasars' spectra.

The two-dimensional spectra of the quasars and the standard stars were obtained using standard IRAF ${ }^{5}$ routines. Each of the objects was initially pair subtracted in order to remove most of

\footnotetext{
4 This redshift interval ensures spectral coverage also of the $\mathrm{H} \alpha$ emission line in the $K$ band

5 The Image Reduction and Analysis Facility (IRAF) is distributed by the National Optical Astronomy Observatory, which is operated by AURA, Inc., under cooperative agreement with the National Science Foundation.
}

the background noise. Then, both the positive and negative residual peaks were analyzed and averaged together. During the analysis, wavelength calibration was achieved using argon arc lamps. The hydrogen features in each standard star were removed prior to removing the telluric features from the quasars' spectra.

Removal of the telluric features and the instrumental response from the quasar spectra was done by dividing these spectra by their respective standard star spectra. Then, any remaining cosmic ray signatures on the quasar spectra were carefully removed. Final, flux-calibrated, quasar spectra were obtained by multiplying these data by blackbody curves with temperatures corresponding to the spectral types of the telluric standards and by a constant factor that was determined by comparing the $H$, for $2.15<z<2.65$, or $K$, for $3.20<$ $z<3.70$, band magnitudes from the Two Micron All Sky Survey (2MASS; Skrutskie et al. 2006) to the integrated flux across the respective band using the flux conversion factors from Table A.2 of Bessell et al. (1998). We do not rely on the telluric standards for the purpose of flux calibration given the relatively narrow slit and the differences in atmospheric conditions between the observations of the quasars and their respective standard stars. For each source, we utilized their SDSS spectrum to verify that the combined SDSS and UKIRT spectra are consistent with a typical quasar optical-UV continuum of the form $f_{\nu} \propto \nu^{-0.5}$ (Vanden Berk et al. 2001). By comparing the flux densities at the rest-frame wavelength of $5100 \AA$ to the flux densities at the rest-frame wavelength in the region of 2000-3500 , dependent on the redshift, in the SDSS spectrum of each source, we verified that the differences 
Table 2

Spectral Measurements of the $\mathrm{H} \beta$ Region and $\mathrm{H} \alpha$

\begin{tabular}{|c|c|c|c|c|c|c|c|c|c|}
\hline $\begin{array}{l}\text { Quasar } \\
\text { (1) }\end{array}$ & $\begin{array}{c}\mathrm{FWHM}_{\mathrm{H} \beta} \\
\left(\mathrm{km} \mathrm{s}^{-1}\right) \\
(2)\end{array}$ & $\begin{array}{c}\mathrm{EW}_{\mathrm{H} \beta} \\
(\AA) \\
(3)\end{array}$ & $\begin{array}{c}\lambda_{\text {peak H } \beta} \\
(\AA) \\
(4)\end{array}$ & $\begin{array}{c}\mathrm{FWHM}_{[\mathrm{O} \mathrm{III}]} \\
\left(\mathrm{km} \mathrm{s}^{-1}\right) \\
(5)\end{array}$ & $\begin{array}{c}\left.\mathrm{EW}_{[\mathrm{O}} \mathrm{III}\right] \\
(\AA) \\
(6)\end{array}$ & $\begin{array}{c}\lambda_{\text {peak[O III] }}{ }^{\mathrm{a}} \\
(\AA) \\
(7)\end{array}$ & $\begin{array}{c}\mathrm{FWHM}_{\mathrm{H} \alpha} \\
\left(\mathrm{km} \mathrm{s}^{-1}\right) \\
(8)\end{array}$ & $\begin{array}{c}\mathrm{EW}_{\mathrm{H} \alpha} \\
(\AA) \\
(9)\end{array}$ & $\begin{array}{c}\lambda_{\text {peakH } \alpha} \\
(\AA) \\
(10)\end{array}$ \\
\hline SDSS J013435.67-093102.9 & 4438 & 99.7 & 15656 & 1625 & 14.6 & 16091 & 2882 & 444 & 21125 \\
\hline SDSS J014850.64-090712.8 & 4716 & 33.7 & 21035 & 1513 & 4.3 & 21680 & $\cdots$ & $\cdots$ & $\cdots$ \\
\hline SDSS J073607.63+220758.9 & 6876 & 94.3 & 21625 & 1640 & 31.6 & 22256 & $\cdots$ & $\cdots$ & $\cdots$ \\
\hline SDSS J142243.02+441721.2 & 4563 & 39.9 & 22607 & $\cdots$ & $\ldots$ & $\cdots$ & $\cdots$ & $\cdots$ & $\cdots$ \\
\hline SDSS J153750.10+201035.7 & 5107 & 69.5 & 21516 & 1613 & 14.6 & 22094 & $\cdots$ & $\cdots$ & $\cdots$ \\
\hline SDSS J153830.55+085517.0 & 5512 & 70.8 & 22161 & 3192 & 26.1 & 22782 & $\cdots$ & $\cdots$ & $\cdots$ \\
\hline SDSS J154359.43+535903.1 & 8301 & 54.3 & 16495 & 1835 & 28.6 & 16843 & 7495 & 543 & 22171 \\
\hline SDSS J154446.33+412035.7 & 7235 & 132.4 & 22202 & $\cdots$ & $\cdots$ & $\cdots$ & $\cdots$ & $\cdots$ & $\cdots$ \\
\hline SDSS J154938.71+124509.1 & 5495 & 42.4 & 16408 & 1544 & 15.4 & 16866 & 5550 & 374 & 22139 \\
\hline SDSS J155013.64+200154.5 & 6539 & 61.9 & 15544 & 1325 & 7.5 & 15960 & 5178 & 391 & 20962 \\
\hline SDSS J160222.72+084538.4 & 6676 & 122.3 & 15951 & 2387 & 19.5 & 16398 & 5629 & 586 & 21517 \\
\hline SDSS J163300.13+362904.8 & 4876 & 57.8 & 22297 & 3768 & 24.6 & 22884 & $\cdots$ & $\cdots$ & $\cdots$ \\
\hline SDSS J165137.52+400218.9 & 4405 & 65.6 & 16234 & 957.8 & 18.5 & 16713 & 4380 & 377 & 21920 \\
\hline SDSS J172237.85+385951.8 & 5938 & 67.9 & 21300 & 3028 & 13.9 & 21866 & $\cdots$ & $\cdots$ & $\cdots$ \\
\hline SDSS J210524.47+000407.3 & 5331 & 25.3 & 16256 & $\cdots$ & $\cdots$ & $\cdots$ & 4530 & 281 & 21975 \\
\hline SDSS J212329.46-005052.9 & 4500 & 48.1 & 15929 & $\cdots$ & $\cdots$ & $\cdots$ & 4084 & 319 & 21540 \\
\hline SDSS J221506.02+151208.5 & 4059 & 100.0 & 20840 & 956.9 & 61.7 & 21450 & $\cdots$ & $\cdots$ & $\cdots$ \\
\hline SDSS J235808.54+012507.2 & 3702 & 63.3 & 21397 & 2652 & 11.6 & 21974 & $\cdots$ & $\cdots$ & $\cdots$ \\
\hline
\end{tabular}

Notes.

${ }^{\text {a }}$ Corresponding to the [O III] $\lambda 5007$ component.

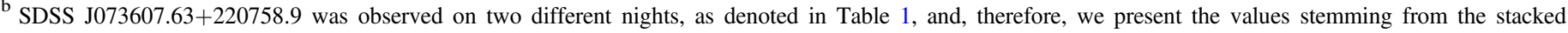
spectrum.

between the two values were within $30 \%$, indicating, at most, only modest flux variations. Such variations, over a temporal baseline of $\sim 6 \mathrm{yr}$ in the rest-frame, are not atypical for such luminous quasars, even if most of these variations are intrinsic as opposed to measurement errors (see, e.g., Kaspi et al. 2007).

\subsection{Fitting of the UKIRT Spectra}

In order to fit the $\mathrm{H} \beta$ and $\mathrm{H} \alpha$ spectral regions, we used a model consisting of a local, linear continuum, which is a good approximation to a power-law continuum given the relatively narrow spectral band, a broadened Boroson \& Green (1992) Fe II emission template, and a multi-Gaussian fit to the emission lines. The Fe II template was broadened by a FWHM value that was free to vary between 2000 and $10000 \mathrm{~km} \mathrm{~s}^{-1}$ and, along with the linear continuum, was removed to more accurately fit the $\mathrm{H} \beta$ and [O III] emission lines. The chosen FWHM to broaden the Fe II template was determined with a least squares analysis.

We fit the $\mathrm{H} \beta$ line using two independent Gaussians, constrained by the width and height of the emission line, simultaneously with one Gaussian for each of the [O III] emission lines. The Gaussians assigned to the [O III] emission lines have identical widths and their intensity ratio was fixed to $I$ $([\mathrm{O}$ III] $\lambda 5007) / I([\mathrm{O}$ III] $\lambda 4959)=3$. The wavelengths of the two [O III] components were fixed to the ratio 5007/4959. For the available $\mathrm{H} \alpha$ features, two Gaussians were fit after a linear continuum was fit and subtracted around the emission line. We do not detect any [N II] emission lines while fitting this region, mainly given our low spectral resolution. The Gaussians were constrained so that the line peak would lie within $1500 \mathrm{~km} \mathrm{~s}^{-1}$ from the wavelength that corresponded to the maximum of the emission line region, the widths could range from 0 to $15,000 \mathrm{~km} \mathrm{~s}^{-1}$, and the flux density was restricted to lie within 0 and twice the maximum value of the emission line.

To estimate the uncertainties on the FWHM and rest-frame equivalent width $(\mathrm{EW})$ of the emission lines, we performed the fitting by adjusting the placement of the continuum according to the noise level in the continuum (see, e.g., Shemmer \& Lieber 2015). Namely, by adjusting the local linear continuum between extremes of the noise around each emission line, we were able to derive an estimate for uncertainties on the FWHM and EW values. For all but two of the sources, the uncertainties on the values of FWHM and $\mathrm{EW}$ in the $\mathrm{H} \beta$ region are on the order of $\sim 5 \%-15 \%$. For SDSS J014850.64-090712.8 and SDSS J163300.13+362904.8, these uncertainties are on the order of $\sim 40 \%$. Similarly, the uncertainties on the FWHM and $\mathrm{EW}$ values for the $\mathrm{H} \alpha$ emission line are up to $\sim 5 \%$.

The uncertainties on the wavelengths of the peaks of all the emission lines are up to $\sim 300 \mathrm{~km} \mathrm{~s}^{-1}$. The majority of this uncertainty arises from the resolution of our spectrograph, however, our choice of a narrow slit was used to combat this. The uncertainty introduced from the pixel-wavelength calibration is minimal, averaging $\sim 5 \mathrm{~km} \mathrm{~s}^{-1}$. The narrow [O III] $\lambda 5007$ emission line provided our most accurate redshift estimates, having uncertainties on wavelength measurements averaging $\sim 150 \mathrm{~km} \mathrm{~s}^{-1}$. The wavelength uncertainties were determined by evaluating our $\mathrm{S} / \mathrm{N}$ and repeated measurements of each of the emission lines.

Basic spectral properties resulting from those fits are reported in Table 2. Columns (2), (3), and (4) provide the FWHM, EW, and the observed-frame wavelength of the peak $\left(\lambda_{\text {peak }}\right)$ of the $\mathrm{H} \beta$ line, respectively. Columns (5)-(7) and (8)-(10) provide similar information for the [O III] $\lambda 5007$ and $\mathrm{H} \alpha$ emission lines, respectively. The fits for the $\mathrm{H} \beta$ and [O III] emission lines appear in Figure 1, and the fits for the $\mathrm{H} \alpha$ emission line appear in Figure 2. 

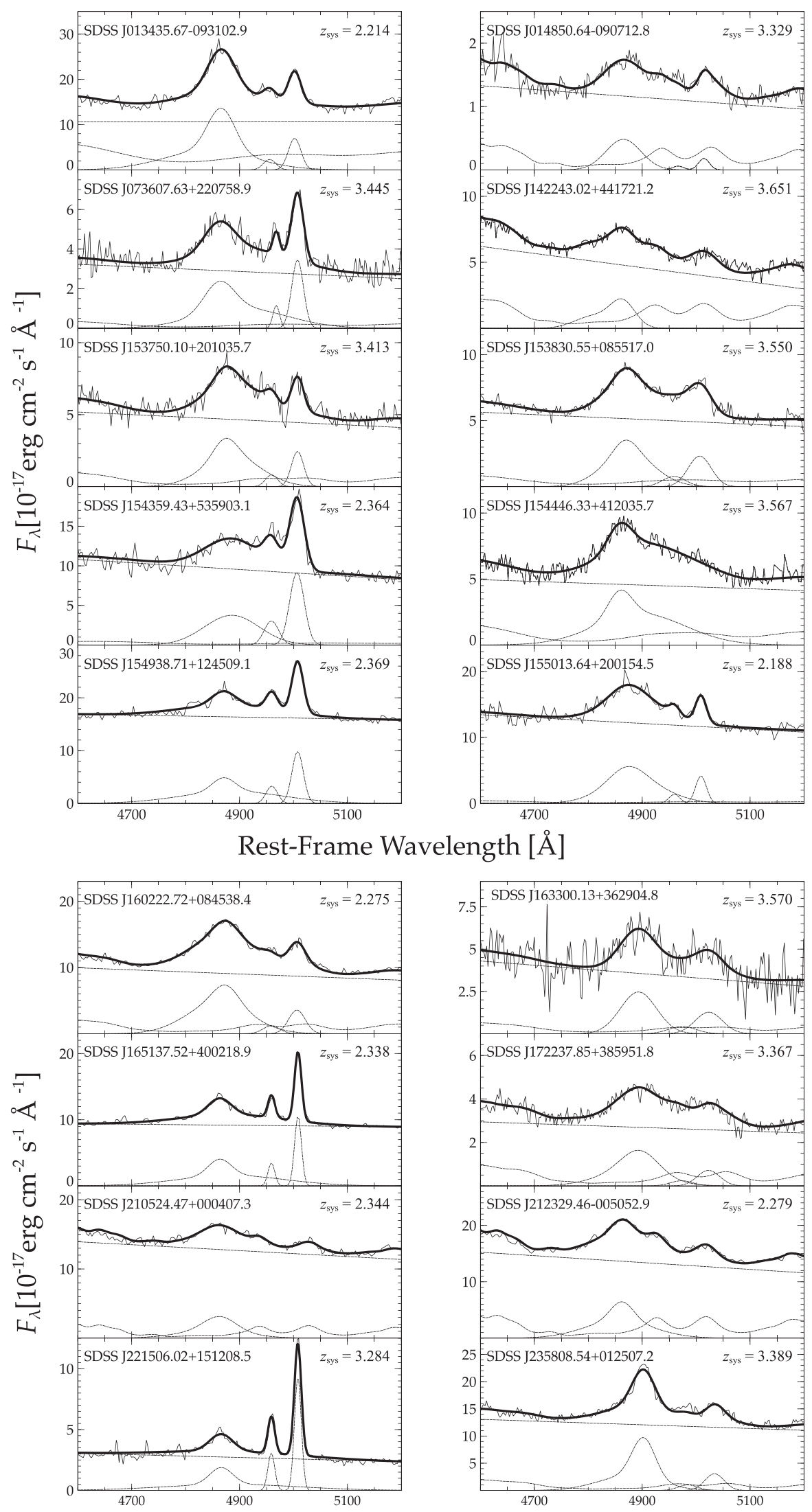

\section{Rest-Frame Wavelength $[\AA]$}

Figure 1. NIR spectra of $2.15<z<3.70$ quasars. The spectrum in each panel is given by a thin solid line. The fit to each individual feature, Fe II, H $\beta$, and [O III] where applicable, and the linear continuum are indicated by dashed lines. The overall fit to each spectrum is given by the bold solid line. 

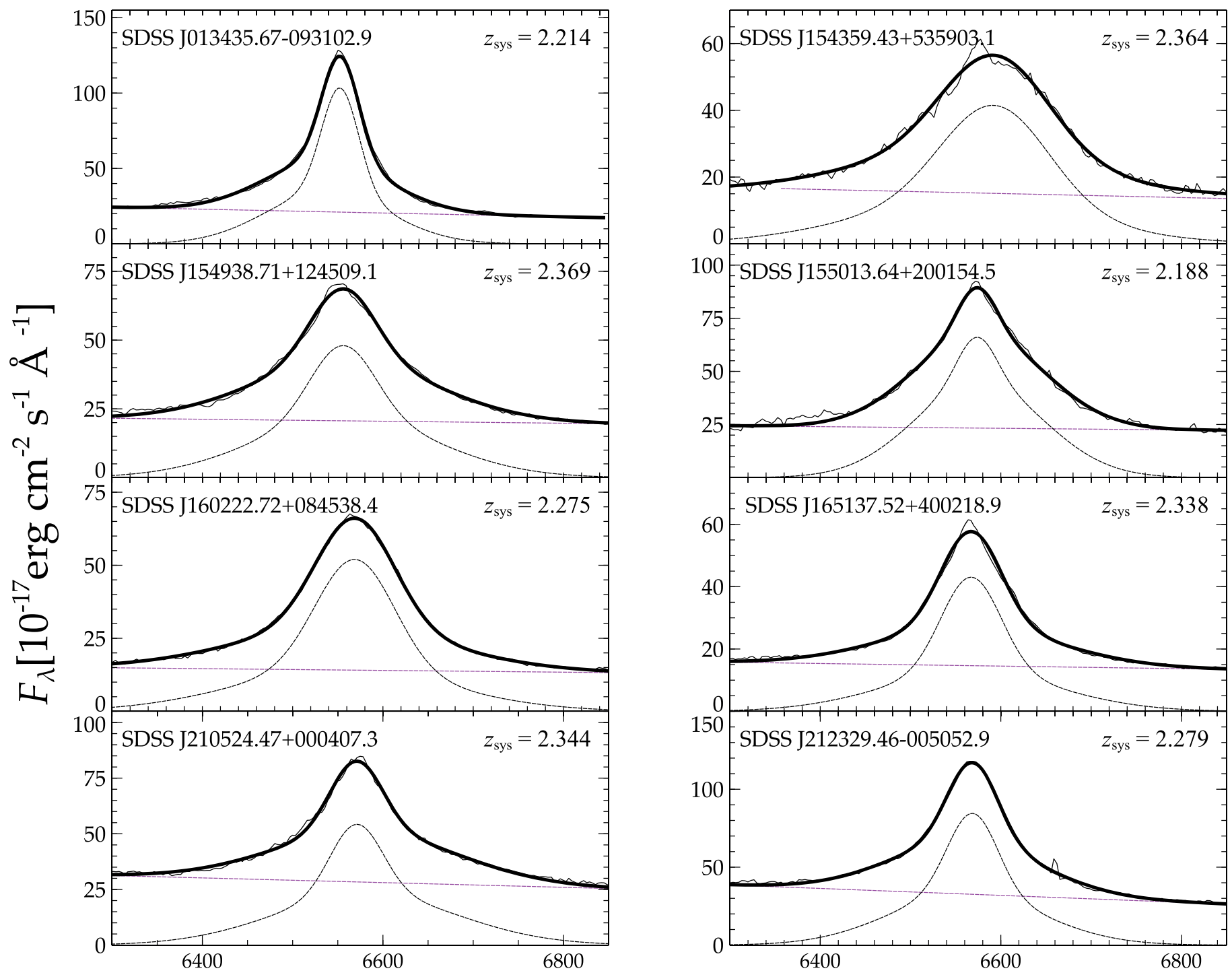

\section{Rest-Frame Wavelength $[\AA]$}

Figure 2. NIR spectra of $2.15<z<2.65$ quasars. The spectrum in each panel is given by a thin solid line. The fit to the H $\alpha$ line and linear continuum are indicated by dashed lines. The overall fit to each spectrum is given by the bold solid line.

\subsection{Spectral Fitting of the CIV Emission Lines}

In order to provide corrections to the UV-based redshifts of our sources, we fit the C IV emission lines present in their SDSS spectra. These fits appear in Figure 3. As suggested in M17, the parameters needed for the correction of the UVbased redshifts are the FWHM and EW of the C IV line, as well as the monochromatic luminosity of the continuum at a restframe wavelength of $1350 \AA$.

The C IV emission line was fit with a local, linear continuum and two independent Gaussians under the same constraints as we report for the $\mathrm{H} \beta$ and $\mathrm{H} \alpha$ emission lines. The spectral properties resulting from this fitting procedure are reported in Table 3 . The uncertainties in each of these measurements were determined by the same method used when evaluating the rest-frame optical emission line uncertainties. Along with this fit, the continuum luminosity, $L_{1350}$, has also been derived by measuring the continuum flux density at rest-frame $\lambda 1350 \AA$ and employing our chosen cosmology. These values also appear in Table 3.

\section{Results}

Combined with the sources in M17, we have a total of 63 objects in our sample, of which, six of our UKIRT objects were excluded from further analysis due to broad absorption line $(B A L)^{6}$ identification: these are noted in Table 1. We then remove an additional BAL quasar, SDSS J014049.18-083942.5, from the sample in M17. Furthermore, we have excluded SDSS J013435.67-093102.9 from our sample given that it is a lensed quasar and its rest-frame UV spectrum is severely attenuated by the foreground lensing galaxy (see, e.g., Ofek et al. 2006). Measurements of the C IV emission line for 52 out of the 55 sources in our combined sample are available in Shen et al. (2011). The C IV FWHM and EW measurements we obtained for 40 of these sources agree to within $\sim 20 \%$ with those from

\footnotetext{
6 Five of these sources are based on BAL quasar identification from Shen et al. (2011). SDSS J073607.63+220758.9 was identified as a BAL quasar following our visual inspection of its SDSS spectrum.
} 
Table 3

Spectral Measurements of C IV

\begin{tabular}{|c|c|c|c|c|c|c|c|}
\hline $\begin{array}{l}\text { Quasar Name } \\
\text { (1) }\end{array}$ & $\begin{array}{c}\mathrm{FWHM}^{\mathrm{a}} \\
\left(\mathrm{km} \mathrm{s}^{-1}\right) \\
(2)\end{array}$ & $\begin{array}{l}\mathrm{EW}^{\mathrm{a}} \\
(\AA) \\
(3)\end{array}$ & $\begin{array}{c}\log L_{1350}{ }^{\mathrm{a}} \\
\left(\mathrm{erg} \mathrm{s}^{-1}\right) \\
(4)\end{array}$ & $\begin{array}{c}\mathrm{FWHM}^{\mathrm{b}} \\
\left(\mathrm{km} \mathrm{s}^{-1}\right) \\
(5)\end{array}$ & $\begin{array}{c}\mathrm{EW}^{\mathrm{b}} \\
(\AA) \\
(6)\end{array}$ & $\begin{array}{c}\log L_{1350}{ }^{\mathrm{b}} \\
\left(\operatorname{erg~\mathrm {s}^{-1}}\right) \\
(7)\end{array}$ & $\begin{array}{c}\lambda_{\text {peak }}{ }^{\mathrm{b}} \\
(\AA) \\
(8)\end{array}$ \\
\hline SDSS J013435.67-093102.9 & 1045 & $\ldots$ & $\ldots$ & $\ldots$ & 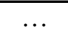 & $\ldots$ & $\ldots$ \\
\hline SDSS J073607.63+220758.9 & $\ldots$ & $\ldots$ & $\ldots$ & 2496 & 10.0 & 46.8 & 6872 \\
\hline SDSS J142243.02+441721.2 & 12475 & 20.8 & 47.0 & 12326 & 17.9 & 47.0 & 7082 \\
\hline SDSS J153750.10+201035.7 & 6080 & 37.9 & 47.1 & 5886 & 33.3 & 47.1 & 6824 \\
\hline SDSS J154446.33+412035. $7^{\mathrm{c}}$ & 15266 & 192.3 & 46.3 & 7350 & 34.4 & 46.6 & 7001 \\
\hline SDSS J154938.71+124509.1 & 4207 & 24.2 & 46.6 & 4740 & 19.6 & 46.5 & 5233 \\
\hline SDSS J155013.64+200154.5 & 4273 & 42.6 & 47.0 & 4858 & 37.4 & 46.9 & 4942 \\
\hline SDSS J160222.72+084538. $4^{\mathrm{c}}$ & 4150 & 27.8 & 47.0 & 5615 & 30.7 & 47.0 & 5065 \\
\hline SDSS J163300.13+362904.8 & 6963 & 34.9 & 46.9 & 6614 & 42.0 & 46.8 & 7067 \\
\hline SDSS J165137.52+400218.9 & 2818 & 49.9 & 46.9 & 2297 & 45.2 & 46.9 & 5172 \\
\hline SDSS J235808.54+012507.2 & $\ldots$ & $\ldots$ & $\ldots$ & 5728 & 20.2 & 47.1 & 6761 \\
\hline
\end{tabular}

Notes.

${ }^{\mathrm{a}}$ Columns (2), (3), and (4) were reported in Shen et al. (2011).

b Columns (5), (6), (7), and (8) were measured from SDSS spectra, as described in the text.

${ }^{\mathrm{c}}$ Indicates broad absorption around the C IV line.

Shen et al. (2011) similarly, measurements of 10 of these sources agree to within $\sim 65 \%$. Generally, these discrepancies are inversely proportional to the $\mathrm{S} / \mathrm{N}$ of the SDSS spectra and are larger in the presence of narrow absorption lines. The spectra for SDSS J025438.36+002132.7 and SDSS J153725.35014650.3 had extremely poor $\mathrm{S} / \mathrm{N}$, resulting in discrepancies of $108 \%$ and $53 \%$ for FWHM, and $57 \%$ and $210 \%$ for EW, respectively, between our measured values and the ones reported in Shen et al. (2011). Substituting our values with the ones reported in Shen et al. (2011) for these objects did not have a significant impact on further analysis.

The observed-frame wavelength of the peak of the C IV emission line was compared to the value predicted by the systemic redshift $\left(z_{\text {sys }}\right)$ to determine the velocity offset of this line. We determine $z_{\text {sys }}$ from the line peak of the emission line with the smallest measurement uncertainty. In order, we take our systemic redshift from [O III] $\left(\sim 50 \mathrm{~km} \mathrm{~s}^{-1}\right), \quad \mathrm{Mg}$ II $\left(\sim 200 \mathrm{~km} \mathrm{~s}^{-1}\right)$ and $\mathrm{H} \beta\left(\sim 400 \mathrm{~km} \mathrm{~s}^{-1}\right)$ (Shen et al. 2016). The C IV velocity offsets are shown and reported in Figure 5 and Table 4, respectively. In Table 4, we also report the redshift measurements provided for these sources in HW10 and Pâris et al. (2018, hereafter P18), where applicable. The velocity offsets introduced from these redshifts with respect to $z_{\text {sys }}$ are presented in Figure 5 and Table 4. In addition to the velocity offsets for the sources in our UKIRT sample, the velocity offsets from Table 1 of M17 have been included in the following regression analysis. The C IV emission line properties for the M17 sample are reported in Table 5.

We note that the $\Delta v_{\mathrm{C}}$ IV values used in M17 differ from the $\Delta v_{\text {C IV }}$ values we compute for the M17 sample since M17 used the $\Delta v_{\mathrm{C}}$ IV values from Shen et al. (2011), combined with the redshift determined from the SDSS pipeline, in order to find $z_{\mathrm{C} \text { IV }}$. Our $\Delta v_{\mathrm{C} \text { IV }}$ values follow directly from the measurement of $\lambda_{\text {peak }}$ (C IV) and our derived $z_{\text {sys. }}$. The origin of the discrepancies between the two velocity offsets used stems from the uncertainty in the $\Delta v_{\mathrm{C} I V}$ values discussed in Shen et al. (2011). The differences between the $\Delta v_{\mathrm{C}}$ IV values we use and those used by M17 are rather small, and using the latter values do not change our results significantly.

A multiple regression analysis has been performed on the velocity offsets and the $\mathrm{C}$ IV emission line properties such that:

$$
\begin{aligned}
\Delta v\left(\mathrm{~km} \mathrm{~s}^{-1}\right)= & \alpha \log _{10}\left(\mathrm{FWHM}_{\mathrm{C} \text { IV }}\right) \\
& +\beta \log _{10}\left(\mathrm{EW}_{\mathrm{C} \text { IV }}\right)+\gamma \log _{10}\left(L_{1350}\right),
\end{aligned}
$$

where $\Delta v$ is the velocity offset and $\alpha, \beta$, and $\gamma$ are the coefficients associated with our regression analysis. The velocity offset created by each redshift derivation method was determined by the following equation

$$
\Delta v=c\left(\frac{z_{\mathrm{meas}}-z_{\mathrm{sys}}}{1+z_{\mathrm{sys}}}\right),
$$

where $z_{\text {meas }}$ is the redshift derived using various methods and reported in the studies indicated below. In order to derive the most reliable redshift correction, four regressions were performed using the following parameters from Equation (1):
1. $\log _{10}\left(\mathrm{FWHM}_{\mathrm{C} \text { IV }}\right), \log _{10}\left(\mathrm{EW}_{\mathrm{C} \text { IV }}\right)$
2. $\log _{10}\left(\mathrm{FWHM}_{\mathrm{C} \text { IV }}\right), \log _{10}\left(L_{1350}\right)$
3. $\log _{10}\left(\mathrm{EW}_{\mathrm{C} \text { IV }}\right), \log _{10}\left(L_{1350}\right)$
4. all three parameters.

In total, this regression analysis was performed on redshifts determined from: (1) the measured line peak of the C IV emission line, (2) HW10, and (3) the SDSS pipeline. The coefficients, errors, and confidence statistics from Equation (1), determined in each of these cases, are reported in Table 6. For the confidence 

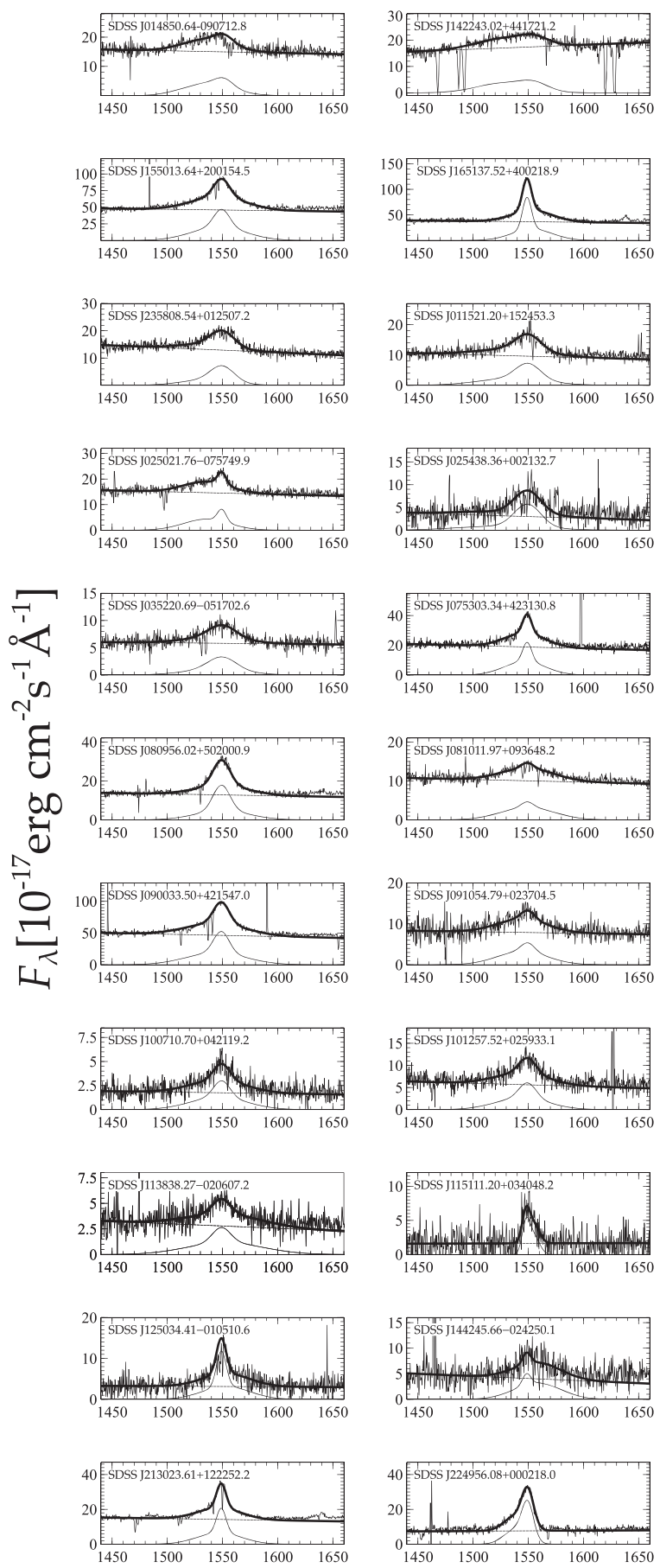
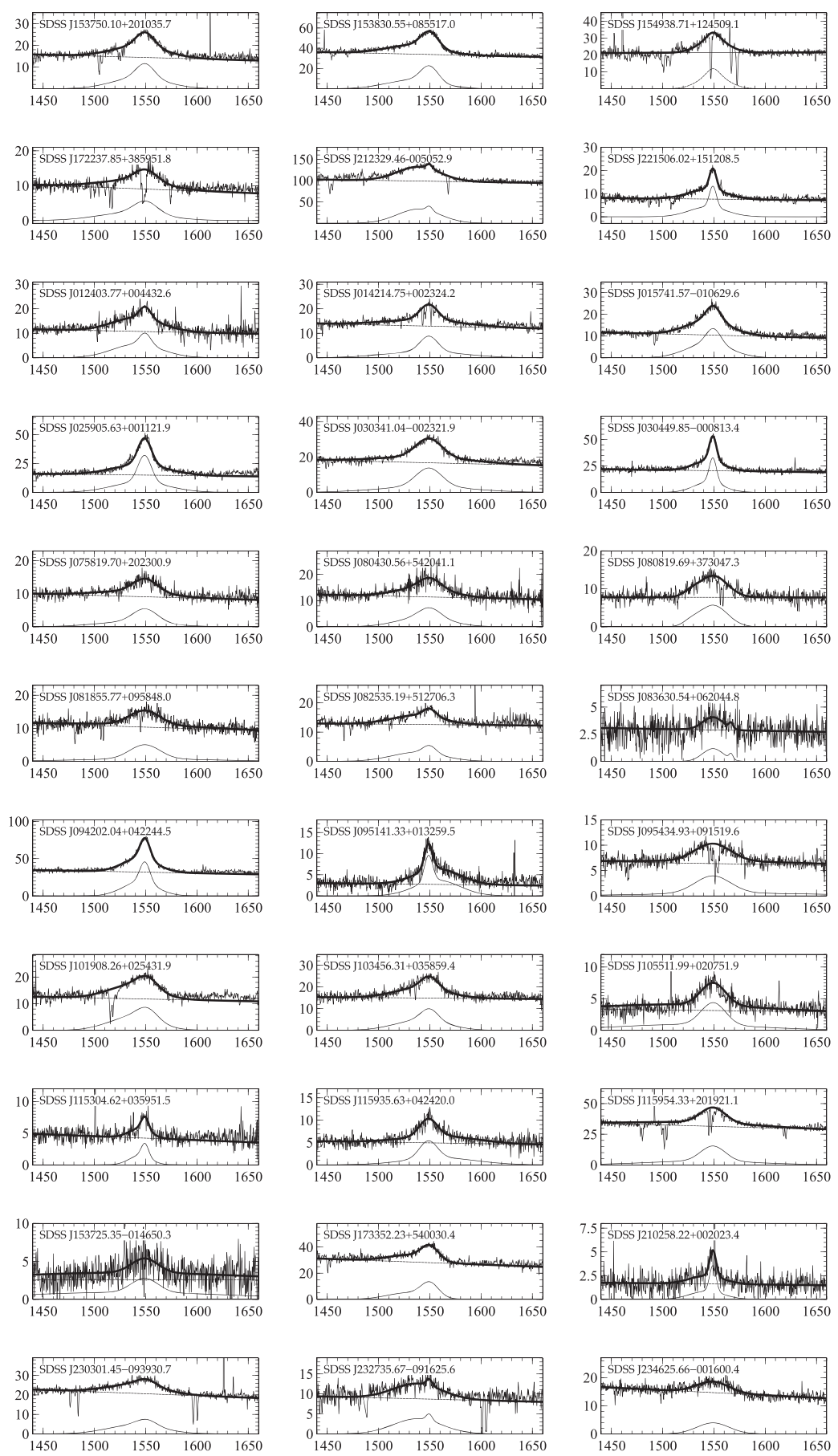

Rest-Frame Wavelength $[\AA]$

Figure 3. C IV fits of all 55 quasars used in the regression analysis. The spectrum and fit to the C IV emission line in each panel are given by a thin solid line. The linear continuum is indicated by a dashed line. The overall fit to each spectrum is given by the bold solid line.

statistics, we report the $t$-value (e.g., Sheskin 2007) to determine the importance of each individual parameter.

The residuals of the velocity offsets after each correction has been determined have been analyzed, and basic statistics resulting from these residuals are listed in Table 7. The residuals before and after correction are presented in Figure 4. The residual distributions show the significant reduction in the velocity offsets before and after each correction. The corrected velocity offsets for C IV- and HW10-based redshifts are closer to zero than the corrected velocity offsets for the SDSS pipeline-based redshift, representative of the larger $\sigma$ value associated with SDSS pipeline redshift estimates. From evaluating the best-fitting coefficients and statistics reported for each correction, we determined the correction that we consider to provide the most reliable results. This correction has been emphasized in bold face in the text. 
Table 4

Redshift Comparison

\begin{tabular}{|c|c|c|c|c|c|c|}
\hline $\begin{array}{l}\text { Quasar } \\
\text { (1) }\end{array}$ & $\begin{array}{c}z_{\text {C IV }}{ }^{a} \\
(2)\end{array}$ & $\begin{array}{c}\Delta v \\
\left(\mathrm{~km} \mathrm{~s}^{-1}\right) \\
(3)\end{array}$ & $\begin{array}{c}z_{\text {pipe }}{ }^{\mathrm{b}} \\
(4)\end{array}$ & $\begin{array}{c}\Delta v \\
\left(\mathrm{~km} \mathrm{~s}^{-1}\right) \\
(5)\end{array}$ & $\begin{array}{c}z_{\mathrm{HW} 10}{ }^{\mathrm{c}} \\
(6)\end{array}$ & $\begin{array}{c}\Delta v \\
\left(\mathrm{~km} \mathrm{~s}^{-1}\right) \\
(7)\end{array}$ \\
\hline SDSS J013435.67-093102.9 & 2.214 & $\ldots$ & $\ldots$ & $\ldots$ & 2.225 & 1029 \\
\hline SDSS J014850.64-090712.8 & 3.274 & -3786 & 3.290 & -2691 & 3.303 & -1796 \\
\hline SDSS J073607.63+220758.9 & 3.436 & -607 & 3.464 & 1285 & $\cdots$ & $\ldots$ \\
\hline SDSS J142243.02+441721.2 & 3.572 & -5097 & 3.397 & -16384 & 3.531 & -7740 \\
\hline SDSS J153750.10+201035.7 & 3.405 & -544 & $\ldots$ & $\ldots$ & $\ldots$ & $\ldots$ \\
\hline SDSS J153830.55+085517.0 & 3.535 & -989 & 3.537 & -856 & 3.563 & 858 \\
\hline SDSS J154359.43+535903.1 & 2.365 & 89 & 2.370 & 536 & 2.379 & 1341 \\
\hline SDSS J154446.33+412035.7 & 3.520 & -3087 & 3.569 & 131 & 3.551 & -1049 \\
\hline SDSS J154938.71+124509.1 & 2.378 & 801 & 2.355 & -1244 & $\ldots$ & $\ldots$ \\
\hline SDSS J155013.64+200154.5 & 2.190 & 188 & 2.194 & 565 & 2.196 & 754 \\
\hline SDSS J160222.72+084538.4 & 2.270 & -458 & $\ldots$ & $\ldots$ & 2.276 & 92 \\
\hline SDSS J163300.13+362904.8 & 3.562 & -525 & 3.538 & -2093 & 3.575 & 328 \\
\hline SDSS J165137.52+400218.9 & 2.339 & 90 & 2.341 & 270 & 2.342 & 360 \\
\hline SDSS J172237.85+385951.8 & 3.350 & -1168 & 3.390 & 1584 & $\ldots$ & $\ldots$ \\
\hline SDSS J210524.47+000407.3 & 2.293 & -4575 & $\ldots$ & $\ldots$ & 2.307 & -3301 \\
\hline SDSS J212329.46-005052.9 & 2.255 & -1376 & 2.233 & -3395 & 2.269 & -92 \\
\hline SDSS J221506.02+151208.5 & 3.285 & 70 & 3.284 & 0 & $\cdots$ & $\cdots$ \\
\hline SDSS J235808.54+012507.2 & 3.366 & -1572 & 3.400 & 753 & $\cdots$ & $\ldots$ \\
\hline
\end{tabular}

Notes.

${ }^{\text {a }}$ Redshifts determined from the $\lambda_{\text {peak }}$ reported in Column (8) of Table 3.

${ }^{b}$ Acquired from P18.

${ }^{c}$ Acquired from HW10.

\subsection{SDSS J142243.02+441721.2 and SDSS J115954.33 $+201921.1$}

SDSS J142243.02+441721.2 from our UKIRT sample has significantly larger velocity offsets compared to the rest of the combined sample. The velocity offsets determined from C IV, HW10, and the SDSS pipeline are $-5097 \mathrm{~km} \mathrm{~s}^{-1}$, $-7740 \mathrm{~km} \mathrm{~s}^{-1}$, and $-16,384 \mathrm{~km} \mathrm{~s}^{-1}$, respectively. The latter velocity offset stems from a misidentification of spectral features in the SDSS spectrum of the source as manifested by the SDSS pipeline products. The SDSS pipeline redshift for this source is $z=3.396$ while the SDSS visual inspection value is $z=3.615$. The disparity between these estimates confirms the misidentification of the emission lines by the SDSS pipeline. Because the velocity offsets for this source had a significant impact on the regression analysis and may be misleading, we have provided the results of the regression analysis with and without this object in Table 7.

The velocity offset of SDSS J115954.33+201921.1 from the M17 sample, with respect to the redshift determined by the SDSS Pipeline is $-10,642 \mathrm{~km} \mathrm{~s}^{-1}$, which is significantly larger than the respective values of the combined sample, excluding SDSS J142243.02+441721.2. SDSS J115954.33+201921.1 was also removed from the SDSS pipeline regression as discussed further in Section 4. Here too, the disparity between the SDSS pipeline redshift value $(z=3.330)$ and the respective visual inspection value $(z=3.425)$ indicates a misidentification of spectral features by the SDSS pipeline.

\section{Discussion}

The results of our multiple regression analysis indicate that the most reliable redshift is obtained by correcting the HW10-based redshift employing the FWHM and EW of the C IV line, the monochromatic luminosity at a rest frame of $1350 \AA$, and the respective coefficients listed under the fourth correction to the HW10 method from Table 6. Using this correction, and removing SDSS J142243.02+441721.2 from the analysis (see Section 3.1), we were able to reduce the uncertainty on the redshift determination from 731 to $543 \mathrm{~km} \mathrm{~s}^{-1}$, yielding an improvement of $\sim 25 \%$ with respect to the HW10-based redshifts; similarly, the mean systematic offset of the redshift determination is reduced from -137 to $+1 \mathrm{~km} \mathrm{~s}^{-1}$ (see Table 7). For comparison, utilizing only the M17 sample of 44 sources, the uncertainty on the HW10-based redshifts is reduced by $\sim 20 \%$. The addition of the five sources from our UKIRT sample that have HW10-based redshifts, comprising a $\sim 10 \%$ increase in the number of sources with respect to the M17 sample, therefore helped to further reduce the uncertainty on the HW10-based redshifts from $\sim 20 \%$ to $\sim 25 \%$. We anticipate that by utilizing a more representative of several hundred high-redshift quasars, we will be able to further improve these uncertainties significantly and the results will become increasingly less biased to small number statistics (e.g., B. Matthews et al. 2020, in preparation).

We note that, when we include the source with the highly discrepant $\Delta v_{\mathrm{C}}$ IV value, SDSS J142243.02+441721.2, in the regression analysis, the best redshift estimates are obtained from the corrected C IV-based redshifts (see Table 7). In this case, the mean systematic redshift offsets reduces from -1023 to $-8 \mathrm{~km} \mathrm{~s}^{-1}$ and the uncertainty on the redshifts' determination decreases from 1135 to $746 \mathrm{~km} \mathrm{~s}^{-1}$ (a 34\% improvement).

As it is apparent, even with this sample of 55 quasars, the methods to determine redshift using rest-frame UV features provide uncertainties as large as $\approx 500-700 \mathrm{~km} \mathrm{~s}^{-1}$. As reported in the first row of each section of Table 7, the uncorrected redshift determinations are significantly inaccurate and imprecise. C IVbased redshifts have a mean systematic offset of $\sim 1000 \mathrm{~km} \mathrm{~s}^{-1}$ (a blueshift) and a similar value for $\sigma$ (the standard deviation). The HW10 method further improves these C IV-based redshifts 
Table 5

C IV Spectral Properties of the M17 Sample

\begin{tabular}{|c|c|c|c|c|c|c|}
\hline $\begin{array}{l}\text { Quasar } \\
\text { (1) }\end{array}$ & $\begin{array}{c}z_{\mathrm{HW} 10} \\
\text { (2) }\end{array}$ & $\begin{array}{l}z_{\text {pipe }} \\
(3)\end{array}$ & $\begin{array}{c}\text { FWHM } \\
\left(\mathrm{km} \mathrm{s}^{-1}\right) \\
(4)\end{array}$ & $\begin{array}{l}\text { EW } \\
(\AA) \\
(5)\end{array}$ & $\begin{array}{c}\log L_{1350} \\
\left(\operatorname{erg~s}^{-1}\right) \\
(6)\end{array}$ & $\begin{array}{c}\lambda_{\text {peak }} \\
(\AA) \\
(7)\end{array}$ \\
\hline SDSS J011521.20+152453.3 & 3.433 & 3.418 & 6236 & 33.3 & 46.6 & 6821 \\
\hline SDSS J014049.18-083942.5 & 3.726 & $\ldots$ & 4635 & 22.7 & 47.2 & 7285 \\
\hline SDSS J014214.75+002324.2 & 3.374 & $\cdots$ & 5013 & 29.2 & 47.0 & 6753 \\
\hline SDSS J015741.57-010629.6 & 3.571 & 3.565 & 5158 & 45.9 & 46.9 & 7049 \\
\hline SDSS J025905.63+001121.9 & 3.377 & 3.372 & 3728 & 65.6 & 46.9 & 6767 \\
\hline SDSS J030341.04-002321.9 & 3.235 & $\ldots$ & 6865 & 41.0 & 47.0 & 6524 \\
\hline SDSS J030449.85-000813.4 & 3.296 & $\cdots$ & 2066 & 27.1 & 47.3 & 6638 \\
\hline SDSS J035220.69-051702.6 & 3.271 & $\cdots$ & 6939 & 24.7 & 46.4 & 6578 \\
\hline SDSS J075303.34+423130.8 & 3.595 & 3.594 & 2804 & 29.4 & 47.3 & 7112 \\
\hline SDSS J075819.70+202300.9 & 3.753 & 3.743 & 6583 & 27.6 & 46.8 & 7333 \\
\hline SDSS J081855.77+095848.0 & 3.688 & 3.692 & 7446 & 26.9 & 47.0 & 7213 \\
\hline SDSS J082535.19+512706.3 & 3.507 & 3.496 & 6839 & 18.7 & 47.1 & 6964 \\
\hline SDSS J083630.54+062044.8 & 3.387 & 3.413 & 5971 & 11.0 & 47.1 & 6767 \\
\hline SDSS J090033.50+421547.0 & 3.294 & 3.296 & 4421 & 40.3 & 47.3 & 6639 \\
\hline SDSS J091054.79+023704.5 & 3.290 & 3.292 & 6184 & 27.7 & 46.4 & 6618 \\
\hline SDSS J094202.04+042244.5 & 3.284 & 3.272 & 3208 & 35.0 & 46.9 & 6617 \\
\hline SDSS J095141.33+013259.5 & 2.419 & 2.425 & 2645 & 96.5 & 46.0 & 5293 \\
\hline SDSS J095434.93+091519.6 & 3.398 & 3.399 & 8671 & 41.1 & 46.7 & 6802 \\
\hline SDSS J100710.70+042119.2 & 2.367 & 2.354 & 4988 & 64.8 & 45.6 & 5199 \\
\hline SDSS J101257.52+025933.1 & 2.441 & 2.436 & 5106 & 39.9 & 46.1 & 5312 \\
\hline SDSS J101908.26+025431.9 & 3.379 & $\ldots$ & 8012 & 34.5 & 47.0 & 6766 \\
\hline SDSS J103456.31+035859.4 & 3.388 & 3.342 & 5972 & 27.8 & 46.8 & 6767 \\
\hline SDSS J105511.99+020751.9 & 3.404 & $\ldots$ & 6372 & 84.5 & 46.1 & 6798 \\
\hline SDSS J173352.23+540030.4 & 3.435 & $\cdots$ & 4994 & 17.1 & 47.4 & 6844 \\
\hline SDSS J210258.22+002023.4 & 3.342 & $\ldots$ & 1733 & 35.0 & 46.8 & 6723 \\
\hline SDSS J213023.61+122252.2 & 3.279 & $\ldots$ & 2596 & 33.6 & 47.0 & 6615 \\
\hline SDSS J224956.08+000218.0 & 3.323 & 3.309 & 2994 & 64.0 & 46.8 & 6677 \\
\hline SDSS J230301.45-093930.7 & 3.470 & $\ldots$ & 8425 & 18.7 & 47.3 & 6898 \\
\hline SDSS J232735.67-091625.6 & 3.470 & $\ldots$ & 8378 & 27.3 & 46.5 & 6582 \\
\hline SDSS J234625.66-001600.4 & 3.281 & $\cdots$ & 7172 & 10.5 & 47.1 & 6892 \\
\hline
\end{tabular}

Note. The $z_{\text {sys }}$ values used in determining the velocity offsets are reported in Column (3) of Table 1 in M17.

${ }^{\mathrm{a}}$ This object was excluded from the regression analysis after visually inspecting its SDSS spectrum and determining it was a BAL quasar.

by reducing the systematic offsets by $\sim 900 \mathrm{~km} \mathrm{~s}^{-1}$ and $\sigma$ by $\sim 300 \mathrm{~km} \mathrm{~s}^{-1}$. Our prescription further reduces the systematic offset by an additional $\sim 100 \mathrm{~km} \mathrm{~s}^{-1}$ and reduces $\sigma$ by an additional $\sim 200-300 \mathrm{~km} \mathrm{~s}^{-1}$. Using the SDSS pipeline redshift estimate, determined from a principal component analysis on multiple features of a spectrum simultaneously (e.g., Bolton et al. 2012), the mean systematic velocity offset for our combined sample is the largest and extends beyond $1000 \mathrm{~km} \mathrm{~s}^{-1}$ with a standard deviation of $1324 \mathrm{~km} \mathrm{~s}^{-1}$. Overall, albeit utilizing a smaller combined sample with respect to the samples we use for C IV- and HW10-based redshifts, the redshifts determined from the SDSS pipeline provide the least reliable results (see Table 7).
Our best correction applied to these redshifts improves the mean systematic velocity offset by $\sim 1000 \mathrm{~km} \mathrm{~s}^{-1}$, similar to the improvement achieved for C IV-based redshifts, but yields only a modest improvement in $\sigma$ which remains large.

In order to test the validity of our method, we have preformed the same regression described in the text on the M17 sources ( $\sim 80 \%$ of our combined sample) and applied it to the remaining sources acquired from UKIRT. The C IV velocity offsets were used in the regression since this sample was the largest of the three UV-based redshift estimates. Prior to correction, the sample of 10 UKIRT sources had a mean, median, and $\sigma$ of $-641 \mathrm{~km} \mathrm{~s}^{-1},-690 \mathrm{~km} \mathrm{~s}^{-1}$, and $952 \mathrm{~km} \mathrm{~s}^{-1}$ 
Table 6

Correction Coefficients

\begin{tabular}{|c|c|c|c|c|c|}
\hline $\begin{array}{l}\text { Correction } \\
\text { (1) }\end{array}$ & $\begin{array}{l}\text { Equation } \\
\text { (2) }\end{array}$ & $\begin{array}{l}\text { Coefficients } \\
\text { (3) }\end{array}$ & $\begin{array}{l}\text { Value } \\
(4)\end{array}$ & $\begin{array}{l}\text { Error } \\
(5)\end{array}$ & $\begin{array}{c}t \text {-value } \\
\text { (6) }\end{array}$ \\
\hline \multirow[t]{9}{*}{$\overline{\mathrm{C} \mathrm{IV}}$} & \multirow{2}{*}{$\alpha \log _{10}\left(\mathrm{FWHM}_{\mathrm{C} \text { IV }}\right)+\beta \log _{10}\left(\mathrm{EW}_{\mathrm{C} \mathrm{IV}}\right)$} & $\alpha$ & -1301 & 195 & -6.68 \\
\hline & & $\beta$ & 2501 & 472 & 5.29 \\
\hline & \multirow[t]{2}{*}{$\alpha \log _{10}\left(\mathrm{FWHM}_{\mathrm{CIV}}\right)+\gamma \log _{10}\left(L_{1350}\right)$} & $\alpha$ & -3966 & 600 & -6.61 \\
\hline & & $\gamma$ & 293 & 48 & 6.14 \\
\hline & \multirow[t]{2}{*}{$\beta \log _{10}\left(\mathrm{EW}_{\mathrm{C} \text { IV }}\right)+\gamma \log _{10}\left(L_{1350}\right)$} & $\beta$ & 2058 & 601 & 3.43 \\
\hline & & $\gamma$ & -88 & 20 & -4.50 \\
\hline & \multirow{3}{*}{$\alpha \log _{10}\left(\mathrm{FWHM}_{\mathrm{C} \mathrm{IV}}\right)+\beta \log _{10}\left(\mathrm{EW}_{\mathrm{C} \mathrm{IV}}\right)+\gamma \log _{10}\left(L_{1350}\right)$} & $\alpha$ & -3670 & 549 & -6.68 \\
\hline & & $\beta$ & 1604 & 450 & 3.57 \\
\hline & & $\gamma$ & 217 & 48 & 4.53 \\
\hline \multirow[t]{9}{*}{ HW10 } & \multirow[t]{2}{*}{$\alpha \log _{10}\left(\mathrm{FWHM}_{\mathrm{C} \mathrm{IV}}\right)+\beta \log _{10}\left(\mathrm{EW}_{\mathrm{C} \mathrm{IV}}\right)$} & $\alpha$ & -1069 & 254 & -4.22 \\
\hline & & $\beta$ & 2517 & 612 & 4.11 \\
\hline & \multirow[t]{2}{*}{$\alpha \log _{10}\left(\mathrm{FWHM}_{\mathrm{CIV}}\right)+\gamma \log _{10}\left(L_{1350}\right)$} & $\alpha$ & -3191 & 869 & -3.67 \\
\hline & & $\gamma$ & 251 & 69 & 3.63 \\
\hline & \multirow[t]{2}{*}{$\beta \log _{10}\left(\mathrm{EW}_{\mathrm{C} \mathrm{IV}}\right)+\gamma \log _{10}\left(L_{1350}\right)$} & $\beta$ & 2219 & 715 & 3.10 \\
\hline & & $\gamma$ & -75 & 24 & -3.18 \\
\hline & \multirow[t]{3}{*}{$\alpha \log _{10}\left(\mathrm{FWHM}_{\mathrm{C} \mathrm{IV}}\right)+\beta \log _{10}\left(\mathrm{EW}_{\mathrm{C} \mathrm{IV}}\right)+\gamma \log _{10}\left(L_{1350}\right)$} & $\alpha$ & -2834 & 819 & -3.46 \\
\hline & & $\beta$ & 1877 & 652 & 2.88 \\
\hline & & $\gamma$ & 161 & 71 & 2.26 \\
\hline \multirow[t]{9}{*}{ SDSS pipe } & \multirow[t]{2}{*}{$\alpha \log _{10}\left(\mathrm{FWHM}_{\mathrm{C} \text { IV }}\right)+\beta \log _{10}\left(\mathrm{EW}_{\mathrm{C} \mathrm{IV}}\right)$} & $\alpha$ & -2380 & 785 & -3.03 \\
\hline & & $\beta$ & 5087 & 1891 & 2.69 \\
\hline & \multirow[t]{2}{*}{$\alpha \log _{10}\left(\mathrm{FWHM}_{\mathrm{C} \mathrm{IV}}\right)+\gamma \log _{10}\left(L_{1350}\right)$} & $\alpha$ & -8024 & 2732 & -2.94 \\
\hline & & $\gamma$ & 613 & 216 & 2.83 \\
\hline & \multirow[t]{2}{*}{$\beta \log _{10}\left(\mathrm{EW}_{\mathrm{CIV}}\right)+\gamma \log _{10}\left(L_{1350}\right)$} & $\beta$ & 4732 & 2240 & 2.11 \\
\hline & & $\gamma$ & -176 & 74 & -2.39 \\
\hline & \multirow[t]{3}{*}{$\alpha \log _{10}\left(\mathrm{FWHM}_{\mathrm{C} \mathrm{IV}}\right)+\beta \log _{10}\left(\mathrm{EW}_{\mathrm{C} \mathrm{IV}}\right)+\gamma \log _{10}\left(L_{1350}\right)$} & $\alpha$ & -6814 & 2830 & -2.41 \\
\hline & & $\beta$ & 3114 & 2212 & 1.41 \\
\hline & & $\gamma$ & 416 & 255 & 1.63 \\
\hline
\end{tabular}

respectively. After running the regression on the M17 sample and applying the new correction to the UKIRT sources, the mean, median, and $\sigma$ improved to $474 \mathrm{~km} \mathrm{~s}^{-1}, 376 \mathrm{~km} \mathrm{~s}^{-1}$, and $772 \mathrm{~km} \mathrm{~s}^{-1}$ respectively, demonstrating the validity of our method.

The SDSS pipeline redshift estimate, as noted in P18, is subject to highly uncertain redshift determinations due to lower $\mathrm{S} / \mathrm{N}$ or unusual objects. As seen in our relatively small sample, large redshift discrepancies are apparent particularly in two of the 39 objects that we have with available SDSS pipeline-based redshifts. In each case, the velocity offsets are $>10^{4} \mathrm{~km} \mathrm{~s}^{-1}$ and, when included in the regression analysis, it nearly tripled the uncertainty on the redshift determination. The most robust redshift determination methods involve a correction based on the C IV spectral properties and UV continuum luminosity to either C IV- or HW10-based redshifts. P18 also provides a redshift based off of visual inspection, $z_{\mathrm{VI}}$. We find that this estimate, where available, provides a much more reliable redshift estimate than the one provided by the SDSS pipeline. The mean systematic offset for this redshift estimate is $-290 \mathrm{~km} \mathrm{~s}^{-1}$ with a standard deviation of $762 \mathrm{~km} \mathrm{~s}^{-1}$.

Regarding the two sources with extremely large velocity offsets, SDSS J142243.02+441721.2 and SDSS J115954.33+201921.1, we note that our best corrections for their UV-based redshifts provide only modest improvements to the redshift determinations, and that their negative velocity offsets (i.e., blueshifts) take on positive velocity offsets (i.e., redshifts), after the correction is applied. The velocity offsets for SDSS J142243.02+441721.2 improve from $-5097 \mathrm{~km} \mathrm{~s}^{-1}$ to $2300 \mathrm{~km} \mathrm{~s}^{-1},-7740 \mathrm{~km} \mathrm{~s}^{-1}$ to $6016 \mathrm{~km} \mathrm{~s}^{-1}$, and $-16,384 \mathrm{~km} \mathrm{~s}^{-1}$ to $11,848 \mathrm{~km} \mathrm{~s}^{-1}$ for C IV-, HW10-, and SDSS pipeline-based redshift estimates, respectively. Similarly, the velocity offsets for SDSS J115954.33+201921.1 changed from $-1264 \mathrm{~km} \mathrm{~s}^{-1}$ to $-58 \mathrm{~km} \mathrm{~s}^{-1}, 407 \mathrm{~km} \mathrm{~s}^{-1}$ to $-656 \mathrm{~km} \mathrm{~s}^{-1}$, and $-10,642 \mathrm{~km} \mathrm{~s}^{-1}$ to $8720 \mathrm{~km} \mathrm{~s}^{-1}$, respectively. While most of the corrected velocity offsets are closer to zero, they do not improve appreciably and still affect the statistics significantly.

The origin for the abnormally large velocity offset of the SDSS pipeline redshift of SDSS J115954.33+201921.1 most likely stems from the misidentification of the emission lines in the SDSS spectra by the SDSS pipeline, as discussed in Section 3.1. As for SDSS J142243.02+441721.2, the origin of the large velocity offset of the C IV-based redshift is intrinsic to the quasar and this should not be confused with the coincidental abnormally large velocity offset stemming from the failure of the SDSS pipeline to correctly identify the UV 
Original

Corrected
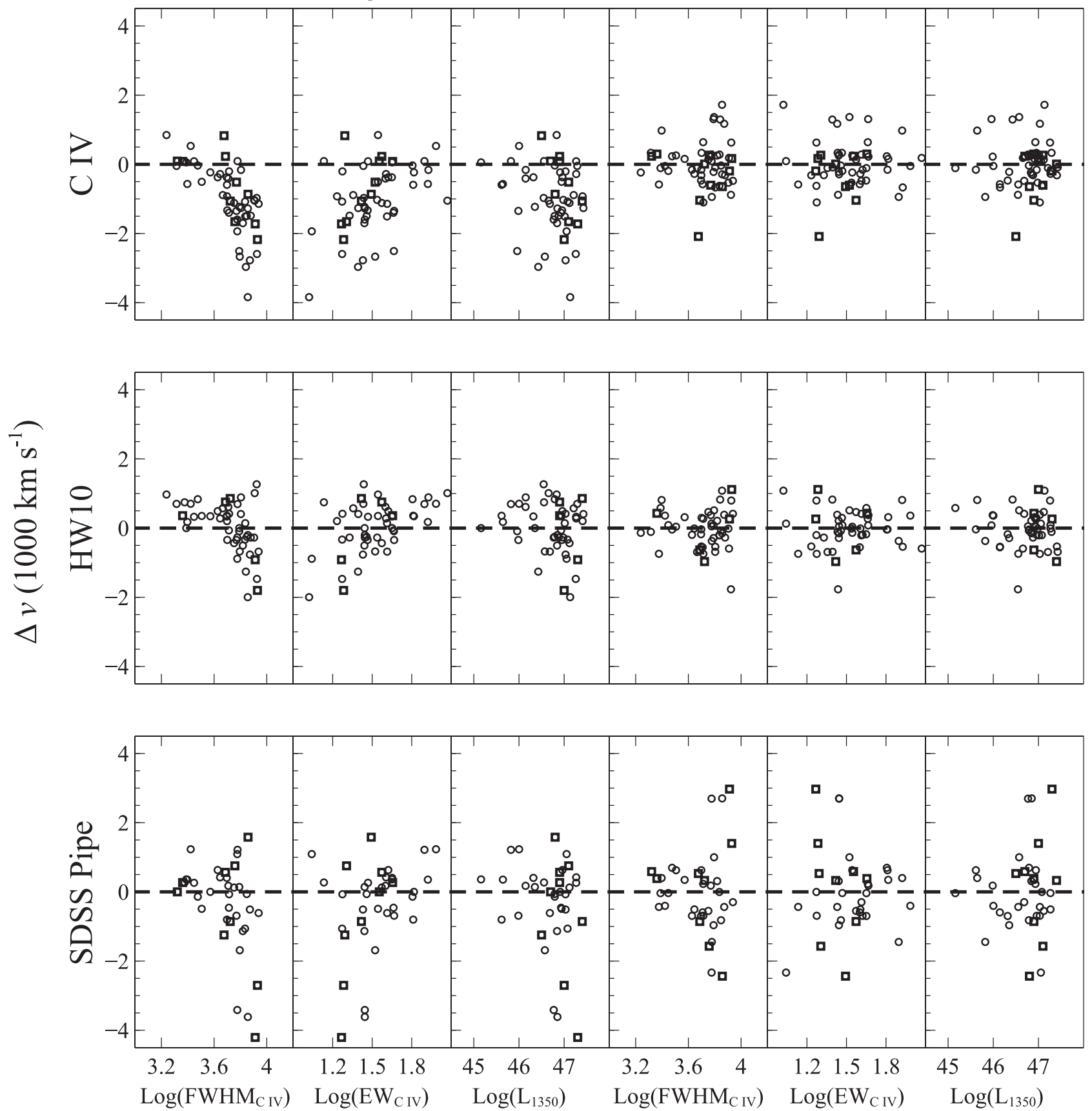

Figure 4. The residual velocity offsets with respect to $z_{\mathrm{sys}}$ before, three leftmost panels, and after, three rightmost panels, correction are presented against our regression parameters. The corrected method displayed refers to Correction 4 without outliers reported in Table 7. Squares (circles) represent data from M17 (UKIRT; this work). The outliers discussed in Section 3.1 do not appear in this plot given their abnormally large velocity offsets.

spectral features (see Section 3.1). Our measured velocity offset of the C IV line $\left(-5097 \mathrm{~km} \mathrm{~s}^{-1}\right)$ is consistent, within the errors, with the value reported in Table 6 of Vietri et al. (2018) for the source $\left(-4670 \mathrm{~km} \mathrm{~s}^{-1}\right)$. Such sources may point to additional spectral parameters that should be taken into account in future prescriptions for UV-based redshift corrections. While such objects may be rare $(\lesssim 5 \%$ in our combined sample), their potential effects on future redshift estimates should be scrutinized to ensure that redshift corrections for the general quasar population are not skewed. The difficulty in correcting the UV-based redshift of SDSS J142243.02+441721.2 is also manifested by the HW10-based redshift which is unable to improve the estimate but rather provides a larger velocity offset $\left(-7740 \mathrm{~km} \mathrm{~s}^{-1}\right)$ with respect to the C IV-based value $\left(-5097 \mathrm{~km} \mathrm{~s}^{-1}\right)$.

With our combined sample of 55 high-redshift quasars, we verify large velocity offsets between UV-based redshift estimates and $z_{\text {sys. }}$. Our calibrations to the UV-based redshift estimates can be used to establish more reliable estimates for $z_{\text {sys }}$ when working with high-redshift quasars in the optical band. This effort will lead to more reliable constraints on a range of measurements that require precise distances for quasars. 


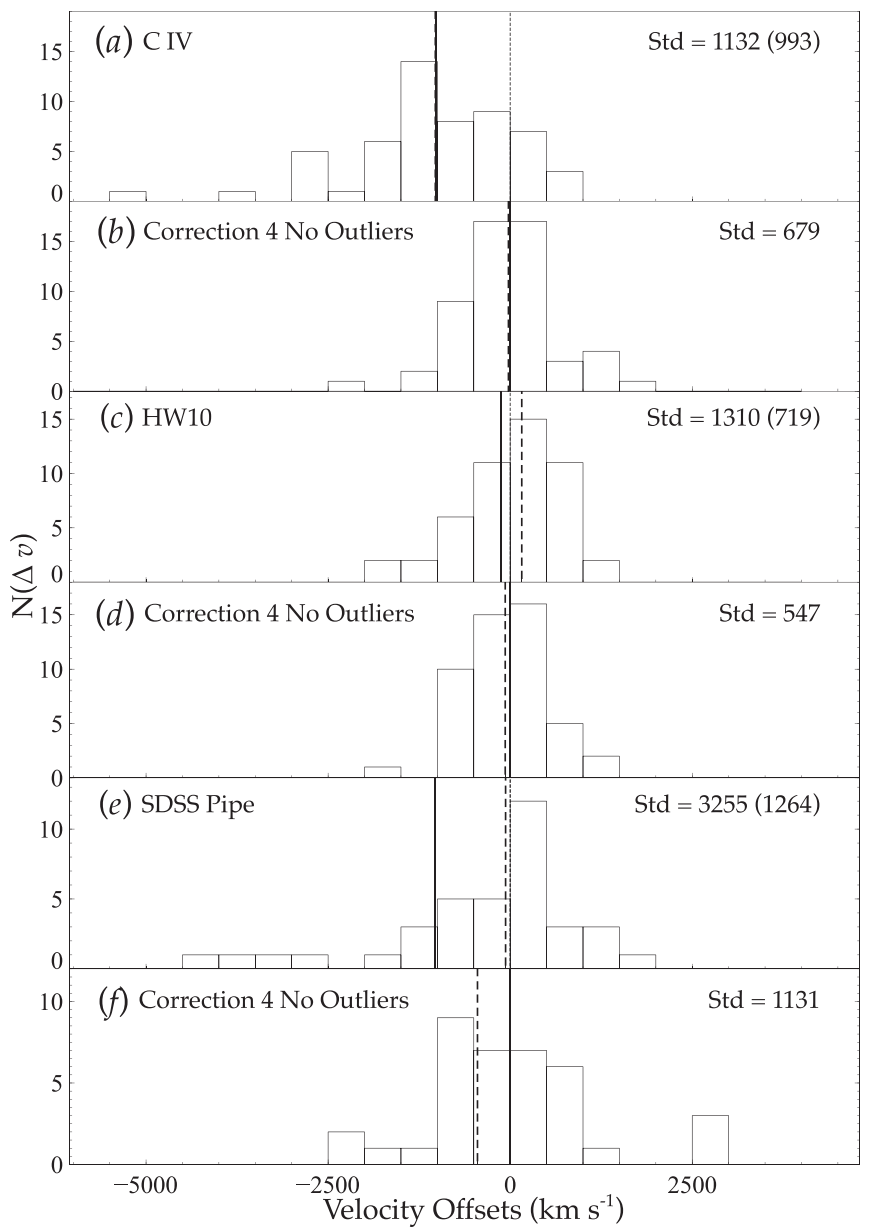

Figure 5. Velocity offsets relative to $z_{\text {sys }}$ before (panels (a), (c), and (e)) and after (panels (b), (d), and (f)) the correction provided in bold face in Table 7. The numbers reported in parentheses are the standard deviations of the original distributions without the outliers. The mean (solid line) and median (dashed line) are marked in each panel. SDSS J142243.02+441721.2 does not appear on the SDSS pipe panel, for clarity, because of its abnormally large velocity offset. The outliers that were removed are discussed in Section 4.

\section{Conclusions}

In the coming decade, $\approx 10^{6}$ high-redshift $(z \gtrsim 0.8)$ quasars will have their redshifts determined through large spectroscopic surveys conducted in the visible band (i.e., rest-frame UV band), e.g., the Dark Energy Spectroscopic Instrument survey (e.g., Levi et al. 2013; DESI Collaboration et al. 2016). Many of these quasars, at $1.5 \lesssim z \lesssim 6.0$, will have the prominent C IV emission line covered in their spectra. The spectral properties of this line can provide a valuable means for correcting UV-based redshifts as we have shown in this work.

Using a sample of 55 quasars, our prescription for correcting UV-based redshifts yields a mean systematic velocity offset which is consistent with zero and further improves the uncertainty on the redshift determination by $\sim 25 \%-35 \%$ with respect to the method of HW10. We also find that UV-based redshifts derived from the SDSS pipeline provide the least reliable results, and the associated uncertainties with respect to $z_{\text {sys }}$ cannot be reduced appreciably. With a larger, uniform sample of high-redshift quasars with NIR spectroscopy (e.g., B. Matthews et al. 2020, in preparation), we plan to improve the reliability of our redshift estimates further and search for
Table 7

Correction Statistics

\begin{tabular}{lcccc}
\hline \hline $\begin{array}{l}\text { Model } \\
(1)\end{array}$ & $\begin{array}{c}\text { Mean } \\
(2)\end{array}$ & $\begin{array}{c}\text { Median } \\
(3)\end{array}$ & $\begin{array}{c}\sigma \\
(4)\end{array}$ & $\begin{array}{c}\text { Skew } \\
(5)\end{array}$ \\
\hline C IV $^{\mathrm{a}}$ & -1016 & -1028 & $1132(993)$ & -1.11 \\
C IV 1 & -20 & -194 & $885(792)$ & 0.55 \\
C IV 2 & -3 & 18 & $837(755)$ & 0.66 \\
C IV 3 & 1 & -80 & $1022(905)$ & 0.67 \\
C IV 4 & $\mathbf{0}$ & $\mathbf{- 2 4}$ & $750^{\mathrm{b}}(679)$ & $\mathbf{0 . 3 7}$ \\
\hline HW 10 & -121 & 159 & $1310(719)$ & -4.09 \\
HW 1 & -14 & -116 & $1123(575)$ & 3.86 \\
HW 2 & -2 & -97 & $1157(638)$ & 3.38 \\
HW 3 & 1 & -73 & $1195(621)$ & 3.98 \\
HW 4 & $\mathbf{1}$ & $-\mathbf{6 8}$ & $1067\left(547^{\mathrm{b}}\right)$ & $\mathbf{3 . 5 9}$ \\
\hline SDSS pipe & -1029 & -63 & $3255(1264)$ & -3.45 \\
Pipe 1 & -31 & -558 & $2954(1161)$ & 2.78 \\
Pipe 2 & -8 & -578 & $2928(1165)$ & 2.66 \\
Pipe 3 & -2 & -697 & $3072(1200)$ & 3.03 \\
Pipe 4 & $-\mathbf{3}$ & $-\mathbf{4 4 9}$ & $\mathbf{2 8 5 1}(\mathbf{1 1 3 1})$ & $\mathbf{2 . 5 4}$ \\
\hline
\end{tabular}

Notes. Bold results are presented in Figure 5. The $\sigma$ reported in parenthesis is the standard deviation once outliers have been removed. For C IV and HW10, only SDSS J142243.02+441721.2 was removed. For SDSS Pipe, SDSS $\mathrm{J} 142243.02+441721.2$ and SDSS J115954.33+201921.1 were removed.

a 55 objects were used in the full correction statistics and 54 objects were used in the correction statistics excluding outliers.

$\mathrm{b}$ The best results, with and without outliers, are further discussed in Section 4.

${ }^{c} 50$ objects were used in the full correction statistics and 49 objects were used in the correction statistics excluding outliers.

d 39 objects were used in the full correction statistics and 37 objects were used in the correction statistics excluding outliers.

additional spectral properties that may further improve these estimates.

We show that the uncertainties on UV-based redshifts for the majority of high-redshift quasars can be reduced considerably by obtaining NIR spectroscopy of a larger sample of sources and using the [O III]-based systemic redshift to inform a C IVbased regression analysis. The reduction in redshift uncertainties is particularly useful for a range of applications involving accurate cosmological distances.

We gratefully acknowledge the financial support by National Science Foundation grants AST-1815281 (C.D., O.S.), and AST-1815645 (M.S.B., A.D.M.). A.D.M. was supported by the Director, Office of Science, Office of High Energy Physics of the U.S. Department of Energy under Contract No. DE-AC0205CH1123 and Award No. DE-SC0019022. We thank the anonymous referee for the constructive report that has improved this manuscript. This research has made use of the NASA/IPAC Extragalactic Database (NED), which is operated by the Jet Propulsion Laboratory, California Institute of Technology, under contract with the National Aeronautics and Space Administration, as well as NASA's Astrophysics Data System Bibliographic Services.

\section{ORCID iDs}

Cooper Dix (1) https://orcid.org/0000-0003-0192-1840 Ohad Shemmer (1) https://orcid.org/0000-0003-4327-1460 Michael S. Brotherton (1) https://orcid.org/0000-0002-1207-0909 Michelle Mason (1i) https://orcid.org/0000-0002-0094-9182 


\section{References}

Bessell, M. S., Castelli, F., \& Plez, B. 1998, A\&A, 333, 231

Bolton, A. S., Schlegel, D. J., Aubourg, É., et al. 2012, AJ, 144, 144

Boroson, T. 2005, AJ, 130, 381

Boroson, T. A., \& Green, R. F. 1992, ApJS, 80, 109

Chen, Z.-F., Qin, Y.-P., Qin, M., et al. 2014, ApJS, 215, 12

DESI Collaboration, Aghamousa, A., Aguilar, J., et al. 2016, arXiv:1611.00036

Font-Ribera, A., Arnau, E., Miralda-Escudé, J., et al. 2013, JCAP, 5, 018

Gaskell, C. M. 1982, ApJ, 263, 79

Gibson, R. R., Jiang, L., Brandt, W. N., et al. 2009, ApJ, 692, 758

Hewett, P. C., \& Wild, V. 2010, MNRAS, 405, 2302

Hogg, D. W. 1999, arXiv:astro-ph/9905116

Hopkins, P. F., \& Elvis, M. 2010, MNRAS, 401, 7

Hutchings, J. B., Cherniawsky, A., Cutri, R. M., et al. 2006, AJ, 131, 680

Kaspi, S., Brandt, W. N., Maoz, D., et al. 2007, ApJ, 659, 997

Levi, M., Bebek, C., Beers, T., et al. 2013, arXiv:1308.0847

Mason, M., Brotherton, M. S., \& Myers, A. 2017, MNRAS, 469, 4675

Ofek, E. O., Maoz, D., Rix, H.-W., et al. 2006, ApJ, 641, 70
Pâris, I., Petitjean, P., Aubourg, É., et al. 2014, A\&A, 563, A54

Pâris, I., Petitjean, P., Aubourg, É, et al. 2018, A\&A, 613, A51

Pâris, I., Petitjean, P., Ross, N. P., et al. 2017, A\&A, 597, A79

Prochaska, J. X., Hennawi, J. F., Lee, K.-G., et al. 2013, ApJ, 776, 136

Richards, G. T., Myers, A. D., Gray, A. G., et al. 2009, ApJS, 180, 67

Schneider, D. P., Richards, G. T., Hall, P. B., et al. 2010, AJ, 139, 2360

Shemmer, Ohad, \& Lieber, Sara 2015, ApJ, 805, 124

Shen, Y., Brandt, W. N., Richards, G. T., et al. 2016, ApJ, 831, 7

Shen, Y., Richards, G. T., Strauss, M. A., et al. 2011, ApJS, 194, 45

Sheskin, D. J. 2007, Handbook of Parametric and Nonparametric Statistical

Procedures (4th ed.; London, Boca Raton, FL: Chapman and Hall, CRC Press)

Skrutskie, M. F., Cutri, R. M., Stiening, R., et al. 2006, AJ, 131, 1163

Spergel, D. N., Bean, R., Doré, O., et al. 2007, ApJS, 170, 377

Tytler, D., \& Fan, X.-M. 1992, ApJS, 79, 1

Vanden Berk, D. E., Richards, G. T., Bauer, A., et al. 2001, AJ, 122, 549

Vietri, G., Piconcelli, E., Bischetti, M., et al. 2018, A\&A, 617, A81

York, D. G., Adelman, J., Anderson, J. E., Jr., et al. 2000, AJ, 120, 1579

Zhao, G.-B., Wang, Y., Saito, S., et al. 2019, MNRAS, 482, 3497 